\title{
Desarrollo empresarial público de derecho privado en el sector del mantenimiento aeronáutico en la Marina de Guerra del Perú
}

CÉSAR Angulo Rebaza ${ }^{1}$

Recibido: 11/11/2020 Aceptado: 19/01/2021 Publicado: 26/07/2021

\begin{abstract}
RESUMEN
La investigación sustenta metodológicamente que es posible desarrollar la industria de mantenimiento aeronáutico en la Marina de Guerra del Perú (MGP) mediante un emprendimiento novedoso en el ámbito de la gestión empresarial al interior de la MGP, es decir, con el diseño de una empresa pública de derecho privado (EPDP), que permita el crecimiento del sector aeronáutico dentro de un marco legal que coadyuve a alcanzar un equilibrio entre eficiencia y eficacia. En el análisis de la situación actual del mantenimiento aeronáutico en la MGP se identificó que no se están aprovechando potencialidades en recursos humanos y materiales certificados (capability). Por ello, la aplicación de teorías administrativas permitirá optimizar el proceso de gestión, lo que generará mayor competitividad y rentabilidad. Se empleó el método descriptivo correlacional a través de la aplicación de encuestas, cuyos resultados validaron la propuesta, así como su viabilidad como órgano descentralizado y especializado del SIMA-Perú. Este escenario contribuiría a satisfacer las necesidades aeronavales de la MGP en las prácticas de gestión de mantenimiento, cultura organizacional, capability del TMA, rentabilidad, agilidad comercial, aseguramiento de la calidad y tecnología, las cuales guardan una relación significativa con el desempeño operacional, para de este modo impactar también en el crecimiento de la tecnología aeronáutica del país.
\end{abstract}

Palabras clave: mantenimiento aeronáutico; rentabilidad; competitividad; capability.

\section{INTRODUCCIÓN}

La competitividad representa un constante desafío empresarial en la búsqueda de eficacia y eficiencia, por lo que los entes gubernamentales y privados necesitan reestructurarse administrativa y financieramente para no perder su propia dinámica. Es el caso del Servicio de Mantenimiento Aeronaval de la Marina de Guerra del Perú (MGP), con certificación de la Dirección General de Aeronáutica Civil (DGAC) como "Organización de Mantenimiento Aprobada (OMA-013): Servicio de Mantenimiento Aeronaval", el cual funciona comercialmente propulsado por la capacidad instalada, certificada y financiada con los fondos que puede aportar la MGP de acuerdo a su disponibilidad, y con la cual no ha podido lograr su autosostenimiento técnico-administrativo y menos cumplir con su misión de mantener la flota aeronaval de la Fuerza de Aviación Naval. Es por esto que se propone el desarrollo de una novedosa gestión empresarial pública en el marco del derecho privado que coadyuve al cumplimiento de la misión y desarrollo aeronáutico de la MGP.

El OMA-013 es el órgano técnico, reparador y de mantenimiento de más alto nivel aeronáutico en la MGP, cuyo objetivo es ejecutar el mantenimiento programado de fabricante (segundo nivel) y reparaciones de componentes (tercer nivel) a las aeronaves asignadas a la Fuerza de Aviación Naval y a las aeronaves y accesorios autorizados en el ámbito de la aviación comercial. Esta organización de mantenimiento aeronáutico ha estado laborando con restricciones presupuestarias desde hace tres décadas y con algunas inversiones representativas no constantes y casi nulas en los últimos cinco años, lo cual ha generado efectos negativos dentro del proceso de mantenimiento, agravados, además, por la carencia de una adecuada gestión administrativa.

Actualmente, el funcionamiento óptimo del OMA-013 está siendo afectado por la creciente actualización de las exigencias

1 Magíster en Ingeniería Aeronáutica por la Universidad Nacional de Ingeniería y magíster en Administración de Empresas por la Universidad Nacional Agraria La Molina (Lima, Perú). Actualmente es piloto marítimo e ingeniero aeronáutico de la Marina de Guerra del Perú, desempeñándose en cargos operacionales, técnicos y administrativos. Cuenta con experiencia liderando proyectos de inversión pública y privada, gestión educativa y gestión industrial (Lima, Perú)

ORCID: https://orcid.org/0000-0002-5261-5598

Autor de correspondencia: cesar.angulo.rebaza.21@gmail.com 
aeronáuticas internacionales por parte de la Dirección General Aeronáutica Civil (DGAC), lo que disminuye su competitividad por la decreciente capacidad técnica certificada de su recurso humano y del material (capability) de sus talleres de mantenimiento aeronáutico (TMA). Este espacio tecnológico no aprovechado perjudica el desarrollo del sector aeronáutico en la MGP, el cual repercute a nivel nacional, debido a que esta actividad todavía no encuentra un desarrollo comercial importante. Por el contrario, la mayoría (más de $80 \%$ ) de las actuales e importantes reparaciones se hacen fuera del país en entidades extranjeras dedicadas a la actividad de ingeniería aeronáutica que aún no llegan a establecerse completamente en el país.

La problemática manifiesta del OMA-013 se encuentra en una situación cada vez más crítica, donde no se pueden aplicar las teorías administrativas y herramientas de gestión necesarias, lo que origina que se deba plantear la siguiente pregunta: ¿Cómo desarrollar una empresa pública de derecho privado competitiva en el negocio del servicio de mantenimiento aeronáutico en la MGP en el Perú?

Ante esa pregunta, esta investigación analiza de manera holística las siguientes características de la organización: infraestructura y recursos humanos (Capability DGAC), rentabilidad y marco legal. Asimismo, analiza las teorías de gestión que pueden ser aplicadas a fin de contribuir a que el OMA-013 sea más competitivo en el servicio de mantenimiento aeronáutico.

Para identificar las condiciones reales del OMA-013 y formular los planes de mejora, es necesario considerar el aporte de diversos autores respecto de los fenómenos que afectan a las organizaciones.

\section{Marco Teórico}

Hamel et al. (2006) señalan que el conocimiento resulta central para el progreso económico de un país, puesto que mejora el desempeño de sus organizaciones. El emprendimiento empresarial interioriza conceptos de sociedades de información, sociedades del conocimiento, trabajadores del conocimiento y sistemas de información en la búsqueda de generar conocimiento explícito y tácito; además, enfatizan como concepto al capital humano, al cual reconocen como lo más valorado para una organización o empresa.

Para Porter (2006), "El campo competitivo es vital para la ventaja competitiva ya que configura la estructura de la cadena de valor, la forma en que se realizan las actividades y la posibilidad de que esas actividades sean compartidas por las unidades de la empresa" (p.44). Además, afirma:

La Estrategia Tecnológica representa entonces para una empresa la política en la que esta decide su desarrollo, mediante el empleo positivo de la tecnología y el poder que tiene el cambio tecnológico para con la estructura del sector industrial en general y aeronáutico en particular, asegurando su ventaja competitiva. (p.133)

La cultura organizacional genera unión y dirección en sus trabajadores, por lo tanto, el trabajo en equipo asegura el éxito de cualquier empresa u organización:

El trabajo en equipo es la capacidad de funcionar con éxito en un entorno de equipo, se está trasladando rápidamente a la primera línea de las habilidades empresariales necesarias (...) La gente que trabaja en grupos operativos, equipos de resolución de problemas, equipos de desarrollo de productos y comités aportan una visión colectiva que multiplica de forma importante su capacidad de innovar. (Blanchard et al., 2006, pp. 70-71)

El trabajo de Vogtlande et al. (2017) analiza las nuevas técnicas en el campo de la ingeniería aeronáutica y evalúa los análisis combinados de costos, valor de mercado y ecocostos, que son la base de la creación de valor ecoeficiente en la remanufactura. Estos autores sostienen que los procesos de remanufactura aeronáutica mejorarán las ventas de aeronaves previamente definidas como exitosas por los usuarios, además de generar valor agregado, nueva vida y nuevas tecnologías.

La remanufactura es el proceso de restauración efectuada por el fabricante a un producto exitoso para ponerlo al nivel de un producto nuevo. Esta tecnología podría ser aprovechada por los talleres de mantenimiento aeronáutico de la MGP, pues representa bajos costos en mano de obra calificada si se compara con los países desarrollados. Además, podría representar un salto en la modernización de la tecnología aeronáutica en el Perú, potenciar la capacidad instalada que posee la Marina y ser una oportunidad de autofinanciamiento.

Davenport (2006) observa y analiza al capital humano y su influencia en la estrategia competitiva. Señala que los trabajadores representan la diferenciación de la empresa y su valor en el mercado en más del $60 \%$ de su valor, lo que resalta la intangibilidad del 
capital humano, el cual es mucho más difícil de conseguir que el capital financiero.

Crespo (2007) señala que el proceso de gestión de mantenimiento condiciona la eficacia y eficiencia de la gestión. La teoría revisada sugiere que la eficacia de las actividades empresariales está enmarcada en términos de capability, rentabilidad, agilidad comercial, tecnología y calidad del servicio bajo la perspectiva del cliente. La eficiencia, siempre seguida de la eficacia, asegura la producción sin desperdicio de recursos, lo que minimiza los costos directos de mantenimiento, y este, a su vez, resulta más competitivo.

Amendola et al. (2015) publican en una fuente abierta sus conclusiones acerca de la gestión de activos. Señalan que la competitividad de las empresas se caracteriza por los constantes recortes de sus presupuestos; por la eficiencia de sus bienes o servicios ante altos estándares de calidad; por el alineamiento a su plan estratégico, por el gasto total (TOTEX, por sus siglas en inglés); por las teorías de inversiones de capital (CAPEX, por sus siglas en inglés), las cuales están en función de la gestión de los activos físicos en la actividad operacional real de la empresa; y, finalmente, por la gestión en el gasto operacional (OPEX), que define los costos asociados a la explotación de estos activos físicos.

Hamel et al. (2006) señalan que es necesario desarrollar un "control estratégico" en el centro de poder y sus respectivas áreas de influencia (filiales). Consideran que algunas decisiones típicas de la estrategia son la tecnología, la definición del mercado de sus productos, énfasis en las diferentes líneas de producto, expansión y diversificación de operaciones, y el deseo de participar en una red global de flujos de productos entre las áreas de influencia o filiales. En ese sentido, la sede principal no solo debe estar interesada en controlar e influenciar todas las decisiones estratégicas, sino también en monitorear su progreso hacia el cumplimiento de las expectativas estratégicas

\section{Marco legal}

El marco legal de la presente investigación se basó en las normas nacionales que resultan ser el cimiento jurídico y de formalización propuesto en este artículo.

Al respecto de la actividad empresarial del Estado peruano, la Ley $\mathrm{N}^{\circ} 24948$ (1988, 2 de diciembre) determina que las empresas de derecho público, las empresas estatales de derecho privado, las empresas de economía mixta y el accionariado del Estado son entidades del sector público nacional, en conformidad con lo establecido por la Constitución Política del Perú, Art. 60 (1993), que reconoce el pluralismo económico sustentado en la coexistencia de diversas formas de empresa y de propiedad. En su artículo 1, la referida ley regula las actividades empresariales del Estado en cuanto a su ámbito; objetivo; organización; funcionamiento; régimen laboral, administrativo, económico y financiero; y evaluación e interacción en los diferentes niveles de gobierno. Asimismo, en su artículo 5 se establece que el Estado genera actividad empresarial de diferentes formas, entre las cuales se destaca, para efectos del presente trabajo, la forma de empresa que se explica luego en el artículo 7:

- Empresas del Estado de derecho privado: Son "las constituidas originalmente o reorganizadas como sociedad anónima de acuerdo a ley, cuyo capital pertenece totalmente al Estado" (Ley N² 24948, 1988).

Adicionalmente, con Decreto Legislativo $\mathrm{N}^{\circ} 1031 \mathrm{se}$ promueve la eficiencia de la actividad empresarial del Estado señalando que, se requiere implementar "nuevas herramientas de gestión y estructuras legales que prioricen los principios de eficiencia, transparencia y gobierno corporativo, entre otros, así como un sistema de control adecuado en un contexto de transparencia" (Decreto Legislativo $\mathrm{N}^{\circ}$ 1031, 2018).

El Fondo Nacional de Financiamiento de la Actividad Empresarial del Estado (FONAFE) es el ente encargado de normar la actividad empresarial del Estado, procurando que estas empresas se gestionen de manera moderna y según reconocidos estándares de calidad de sus productos (bienes y/o servicios), de modo que generen valor económico en favor de la sociedad y que respeten el medio ambiente para lograr el sostenido desarrollo del Perú, con base en la innovación e integración como valores específicos corporativos.

De lo expuesto en los párrafos precedentes, se puede apreciar que el Estado peruano tiene la facultad de ejercer actividad empresarial, directa o indirectamente, en las mismas condiciones que una empresa privada, aunque con especiales características en función a su normativa y regulaciones, en las que una empresa pública de derecho privado (EPDP) puede hacer todo lo que no esté prohibido legalmente a fin de optimizar sus procesos de gestión, tal como lo sugiere Mateo (2015), quien en su tesis de doctorado investiga con el objetivo general 
de definir los procesos de gestión de mantenimiento y las causas que afectan al éxito en los modelos de desarrollo.

\section{Servicios Industriales de la Marina, SIMA}

La empresa SIMA es una empresa pública de derecho privado (EPDP) de la Corporación FONAFE, constituida como sociedad anónima en 1999, creada con el objetivo de "promover y desarrollar la industria naval, industrias complementarias y conexas", dando prioridad a la reparación y construcción de los buques de la MGP (SIMA, 2000). En el contexto actual, tiene la potencialidad de desarrollar la actividad en el ámbito aeronaval como rubro de negocio con la integración del capability desarrollado por el OMA-013.

De acuerdo con el portal del SIMA (2020), la actividad empresarial del Estado se encuentra regulada por la Ley $N^{\circ} 24948$ (1988), la cual, en su artículo 6 señala que "Son empresas de derecho público las que se crean por ley y gozan de atributos propios de la administración pública", y en su artículo 7 señala que "Son empresas de Derecho Privado las constituidas originalmente o reorganizadas como sociedad anónima de acuerdo a ley, cuyo capital pertenece totalmente al Estado".

En ese sentido, el marco legal ha sido favorable para el desarrollo del SIMA y ha permitido que cuente con tres centros de operaciones ubicados en el Callao y sus filiales en Chimbote e Iquitos, siendo sus tres principales líneas de negocio:

- Mantenimiento preventivo y correctivo a naves, denominado reparaciones navales.

- Construcción de embarcaciones, denominado construcciones navales.

- Producción de estructuras metálicas (puentes, torres, compuertas hidroeléctricas, etc.), denominada metalmecánica.

En su tesis de doctorado, Villalón (2015) concluye en que es necesario desarrollar y aplicar una gran variedad de métodos cuantitativos y cualitativos en la búsqueda efectiva de conocer las competencias de gestión y esquemas de vinculación que permitan construir un modelo que genere un alto desempeño. Al respecto, se puede afirmar que SIMA es una empresa concentrada en el ámbito de la ingeniería naval, que alberga actividad aeronaval en sus plataformas navales, que se encuentra diversificada en importantes actividades tecnológicas, que cuenta con excelentes competencias para la gestión técnica y empresarial, y que genera altos niveles de desempeño, únicos en la nación, con lo que contribuye al desarrollo del país.

Por esta razón, al analizar el marco legal de la teoría expuesta, la EPDP resulta ser un modelo de gestión empresarial que se caracteriza por objetivos a largo plazo, satisfacción e impacto positivo en el desarrollo de la nación. Este modelo de gestión provee herramientas de gerencia moderna que generan reputación y valor a una empresa ante sus stakeholders y las incorpora a los procesos de gestión desde una perspectiva sistémica, lo cual se reflejaría en la generación de valor público-privado. Lo planteado genera una enorme oportunidad para el desarrollo empresarial en el servicio de mantenimiento aeronaval en la búsqueda de desarrollo social, tecnológico, empresarial y de la defensa interna y externa de la nación.

De acuerdo a las teorías presentadas y la experiencia de la MGP en el desarrollo aeronáutico del país, la presente investigación plantea que el Servicio de Mantenimiento Aeronaval OMA-13 tendría éxito empresarial como EPDP soportándose en el marco legal del SIMA, como filial del desarrollo de la capacidad técnica del medio aeronaval bajo su responsabilidad legal, sin necesidad de crear otra entidad que se gestione como EPDP. De esta manera se fomentaría el desarrollo de la tecnología en el país, puesto que no existe tal desarrollo tecnológico especializado y particular en el ámbito naval ni aeronaval.

Análisis FODA de las variables según sus dimensiones validadas y sus resultados estadísticos

Riquelme (2016), en su ilustración sobre análisis y planeamiento estratégico, explica que el término FODA es un acróstico de:

- Fortalezas: factores críticos positivos con los que se cuenta;

- Oportunidades: aspectos positivos que se pueden aprovechar utilizando las fortalezas;

- Debilidades: factores críticos negativos que se deben eliminar o reducir; y

- Amenazas: aspectos negativos externos que podrían obstaculizar el logro de nuestros objetivos.

El análisis FODA resulta altamente recomendable, pues es la etapa previa a la toma de decisiones. Este método sencillo y eficaz sirve para determinar la condición real de una empresa en cuanto a su 
entorno, con lo cual puede planear una estrategia con visión de futuro.

Con el objetivo de desarrollar una EPDP competitiva en beneficio de la MGP y del país en el sector aeronáutico, se realizó, de acuerdo con la teoría presentada, un análisis de fortalezas, oportunidades, debilidades y amenazas (FODA), el cual determinó, cualitativamente y según ponderados cuantitativos, las estrategias a tomar según objetivos estratégicos a cumplir (decisión estratégica). Este análisis presentó la situación real del OMA-013, que se encuentra en una "área de vulnerabilidad", lo cual, en base al trabajo de campo y experiencia en la gestión de esta organización de mantenimiento, es relevante mencionar.

El análisis FODA presentó a un OMA-013 incapaz de autosostenerse, fundamentalmente por no ser competitivo en el sector aeronáutico, y cuya principal debilidad es el tiempo en lograr que las aeronaves de sus clientes estén en línea de vuelo y operando, debido a las reglas de la administración pública en la que se desenvuelve, lo cual se agrava por la repercusión que tiene el recurso financiero disponible para lograr estándares de capability exigidos y reglamentados por la DGAC.

En la Tabla 1 se muestra una matriz FODA del OMA013. Este enfoque dará a la gerencia elementos muy importantes para construir una estrategia de futuro que permitirá desarrollar acciones concretas enfocadas a mejorar la competitividad y posición en el mercado.

Con base en esta realidad, se planteó la dirección de la gestión del OMA-013 como EPDP a través de estrategias a futuro tomando en consideración la verificación de la encuesta planteada en la investigación, la cual determinó las estrategias a implementar en el Servicio de Mantenimiento Aeronaval de acuerdo con los valores obtenidos y en función a los objetivos planteados en la Tabla 2 .

El análisis interno y externo del entorno en el que se desenvuelven las actividades del OMA-013 concluye en que se requieren estrategias que redefinan su actividad como EPDP con el objetivo de obtener mayor

Tabla 1. Matriz FODA OMA-013.

\begin{tabular}{|c|c|}
\hline FACTORES INTERNOS & FACTORES EXTERNOS \\
\hline $\begin{array}{l}\text { FORTALEZAS } \\
\text { - RR. HH. certificado en el servicio. } \\
\text { - } \quad \text { Renovación de RR. HH. certificado. } \\
\text { - } \quad \text { Aumento de clientes medianos y grandes. } \\
\text { - } \quad \text { Capacidad instalada diferenciada. } \\
\text { - } \quad \text { Demanda en aumento asegurado. }\end{array}$ & $\begin{array}{l}\text { OPORTUNIDADES } \\
\text { - Incremento de O/S y presupuesto asociado. } \\
\text { - Déficit de talleres de mantenimiento aeronáutico en el } \\
\text { mercado. } \\
\text { - Ampliación de capabilities del TMA (más O/S). } \\
\text { - Proveedores logísticos confiables. }\end{array}$ \\
\hline $\begin{array}{l}\text { DEBILIDADES } \\
\text { - } \text { Costos de upgrade tecnológico y RR. HH. } \\
\text { - Inversión continua en capability. } \\
\text { - } \quad \text { Mediana capacidad retención de RR. HH. } \\
\text { - } \quad \text { Poca agresividad en marketing. } \\
\text { - } \quad \text { Falta de incentivos a RR. HH. clave inicio. }\end{array}$ & $\begin{array}{l}\text { AMENAZAS } \\
\text { - Aparición de más competencia asociada. } \\
\text { - } \text { Fortalecimiento otros TMA de similar función. } \\
\text { - Proveedores logísticos no confiables. } \\
\text { - Capacidad de retención de RR. HH. inicio-CG. }\end{array}$ \\
\hline
\end{tabular}

Fuente: Elaboración propia.

Tabla 2. Estrategias y objetivos planteados en la investigación.

\begin{tabular}{|l|l|}
\hline Variables & Estrategia / Objetivo \\
\hline \multirow{2}{*}{$\begin{array}{l}\text { Independiente: } \\
\text { Desarrollo del Capability del OMA-13 }\end{array}$} & Gestión de mantenimiento (UNE-EN 15341) \\
\cline { 2 - 2 } & Cultura organizacional \\
\cline { 2 - 2 } $\begin{array}{l}\text { Dependiente: } \\
\text { Competitividad }\end{array}$ & Capability TMA \\
\cline { 2 - 2 } & Rentabilidad \\
\cline { 2 - 2 } & Agilidad comercial \\
\cline { 2 - 2 } & Aseguramiento de la calidad \\
\cline { 2 - 2 } & Tecnología \\
\hline
\end{tabular}

Fuente: Elaboración propia. 
competitividad en el mercado nacional y que aseguren que el OMA-013 salga del área de vulnerabilidad en la que se encuentra al no poder cumplir con su misión principal y menos lograr un autosostenimiento financiero que permita cubrir los requerimientos de la MGP y de la nación en el sector aeronáutico.

\section{METODOLOGÍA}

El presente estudio se desarrolló en el marco de una investigación mixta descriptivo-correlacional, del tipo observacional transeccional, que busca determinar el grado de relación existente entre las variables.

De acuerdo con la investigación de campo, efectuada en los talleres de mantenimiento aeronáutico del Servicio de Mantenimiento Aeronáutico (OMA013) de la Marina de Guerra del Perú, se consideraron los siguientes aspectos metodológicos: diseño de la investigación, variables, población, instrumentos, herramientas de recolección y análisis de datos.

En ese sentido, la investigación fue corroborada por datos de carácter cualitativo, producto de una encuesta del tipo escala de Likert, de tal manera que la inferencia pudo ser medible de acuerdo con las variables establecidas en el estudio. Este análisis está relacionado con los métodos inductivos que obtienen registros narrativos de los fenómenos que son estudiados mediante técnicas de observación directa, entrevistas, notas de campo y encuestas a la población de interés.

El tamaño de la muestra abarca a 104 personas dedicadas a la gestión técnica y administrativa de 10 talleres de mantenimiento aeronáutico certificados por la MGP ante la Dirección General de Aeronáutica Civil. Debido a la constante rotación militar, el número de personas fue superior al mínimo determinado en la muestra, de manera que pudiera cubrirse cualquier eventualidad que alterara el estudio, es decir, con la finalidad de conseguir información suficiente para aplicar técnicas estadístico-descriptivas, y que estas fueran aplicadas a las personas que gestionan el OMA-013. El número mínimo de personas a ser encuestadas se estableció al $95 \%$ de confiabilidad.

\section{RESULTADOS Y DISCUSIÓN}

\section{Resultados estadísticos del Servicio de Mante- nimiento Aeronaval, OMA-013}

El instrumento de medición empleado fue el cuestionario, que se aplicó al grupo de control muestral constituido por gerentes, jefes oficiales, oficiales subalternos y jefes de taller del OMA-013. Posterior a los resultados y a la validación correspondiente, se efectuó el respectivo análisis, en el que se determinaron las dimensiones en las que se desenvuelven las variables dependientes e independientes, con los resultados presentados líneas abajo.

\section{Contraste estadístico de la hipótesis principal $\left(\mathrm{H}_{\mathrm{p}}\right)$}

Para analizar y presentar el contraste estadístico de la hipótesis principal $\left(\mathrm{H}_{\mathrm{p}}\right)$, "Desarrollar al OMA013 como una EPDP en la Marina de Guerra del Perú (MGP) permitirá hacerla competitiva en el servicio de mantenimiento aeronáutico en el Perú", se calcularon por ANOVA el intervalo de confianza y las diferencias significativas de las varianzas de las medias que se quisieron contrastar (pretest y postest). Luego se compararon estadísticamente las diferencias de los valores de las medias calculadas según la hipótesis principal y sus hipótesis secundarias $\left(\mathrm{H}_{\mathrm{s}}\right)$ en los 2 momentos, pretest y postest, a los cuales corresponden; se obtuvo como resultado del proceso del trabajo de campo el convencimiento de la importancia del recurso humano especializado del OMA-013, con el que se trabajaron las variables independientes, lo que resultó una diferencia positiva en las variables dependientes en el postest.

La $H_{p}$ muestra una variable independiente caracterizada por tres dimensiones: gestión de mantenimiento, cultura organizacional y capability de los talleres de mantenimiento aeronáutico; estas, al mejorar su gestión como EPDP, influyen en la competitividad del OMA-013 en el sector aeronáutico. La variable dependiente se caracteriza en cuatro dimensiones: rentabilidad, agilidad comercial, aseguramiento de la calidad y tecnología.

Las encuestas realizadas en el pretest y postest permitieron obtener información importante sobre la valoración que tiene el personal respecto al funcionamiento del OMA-013 y, a través de ello, plantear alternativas para potenciar la capacidad instalada.

En ese sentido, se evaluaron las ponderaciones obtenidas de la encuesta del pretest (Tabla 3) y postest (Tabla 4), prestando primordial atención al grado de significancia al 95\% para esta investigación $(p<0.05)$ para demostrar la hipótesis principal $\left(\mathrm{H}_{\mathrm{p}} / \mathrm{H}_{1}\right)$. 


\section{Significación por magnitud de diferencia entre medias}

La comparación de medias de la variable dependiente se grafica en la Figura 1, donde se aprecia una notable diferencia entre la primera etapa del estudio y la segunda. El incremento del momento postest es debido al grupo poblacional del OMA013, al cual se le aplicó el instrumento de medición validado. Se identificaron plenamente a las variables para la gestión de mantenimiento (rentabilidad, agilidad comercial, aseguramiento de la calidad y tecnología) dentro de un marco legal adecuado (EPDP) como un determinante impulsor del éxito empresarial de esta Organización de Mantenimiento de la MGP.

La diferencia de medias entre el pretest y postest muestra desviaciones típicas debido a sus diferencias. En el análisis de la t muestra aparece el dato estadístico más importante para corroborar la hipótesis y sus variables, es decir, el grado de significancia $(p<0.05)$, por el que se concluye que las características de la EPDP empleada para el desarrollo del OMA-013 generará mayor competitividad en el sector aeronáutico y, por consiguiente, capacidad de autosostenimiento y rentabilidad, al encontrar en el postest un aumento positivo en la ponderación versus los valores que habían alcanzado en el pre test, el cual se aprecia en la Figura 2.

De acuerdo con los resultados del análisis FODA y el análisis estadístico que se practicaron en la investigación, las hipótesis planteadas como guías de la investigación han sido sustentadas a razón de una verdad metodológicamente demostrada, lo cual hace posible la determinación de objetivos estratégicos en un plan de gestión que influya positivamente en mejorar la realidad del sector aeronáutico en el Perú. Las hipótesis planteadas en la investigación recibieron evidencia empírica, las cuales fueron ponderadas según el fenómeno evaluado, mostrando

Tabla 3. Variable dependiente "Competitividad": Datos promedio pretest.

\begin{tabular}{|l|c|c|c|c|c|c|}
\hline \multicolumn{1}{|c|}{ V. Dependiente } & N & Media & Desv. Est. & Q1 & Mediana & Q3 \\
\hline Competitividad & 104 & 3.1524 & 0.602 & 2.8148 & 3.0926 & 3.4074 \\
\hline
\end{tabular}

$\mathrm{N}$ : Tamaño de la muestra.

Fuente: Elaboración propia.

Tabla 4. Variable dependiente "Competitividad": Datos promedio postest.

\begin{tabular}{|l|c|c|c|c|c|c|}
\hline \multicolumn{1}{|c|}{ V. Dependiente } & N & Media & Desv. Est. & Q1 & Mediana & Q3 \\
\hline Competitividad & 104 & 4.5196 & 0.0062 & 4.5185 & 4.5185 & 4.5185 \\
\hline
\end{tabular}

Fuente: Elaboración propia.

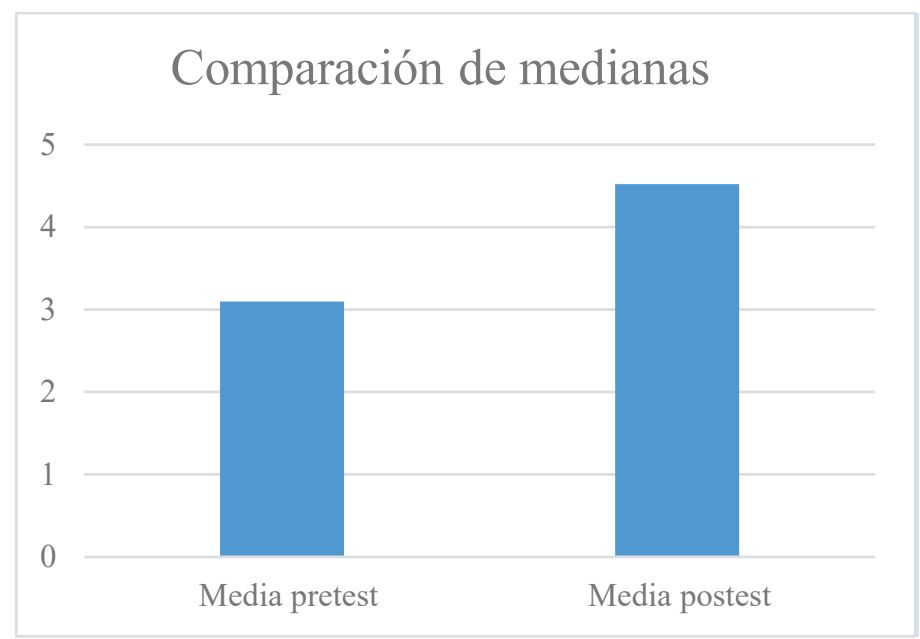

Figura 1. Comparación de medias de variable dependiente.

Fuente: Elaboración propia. 


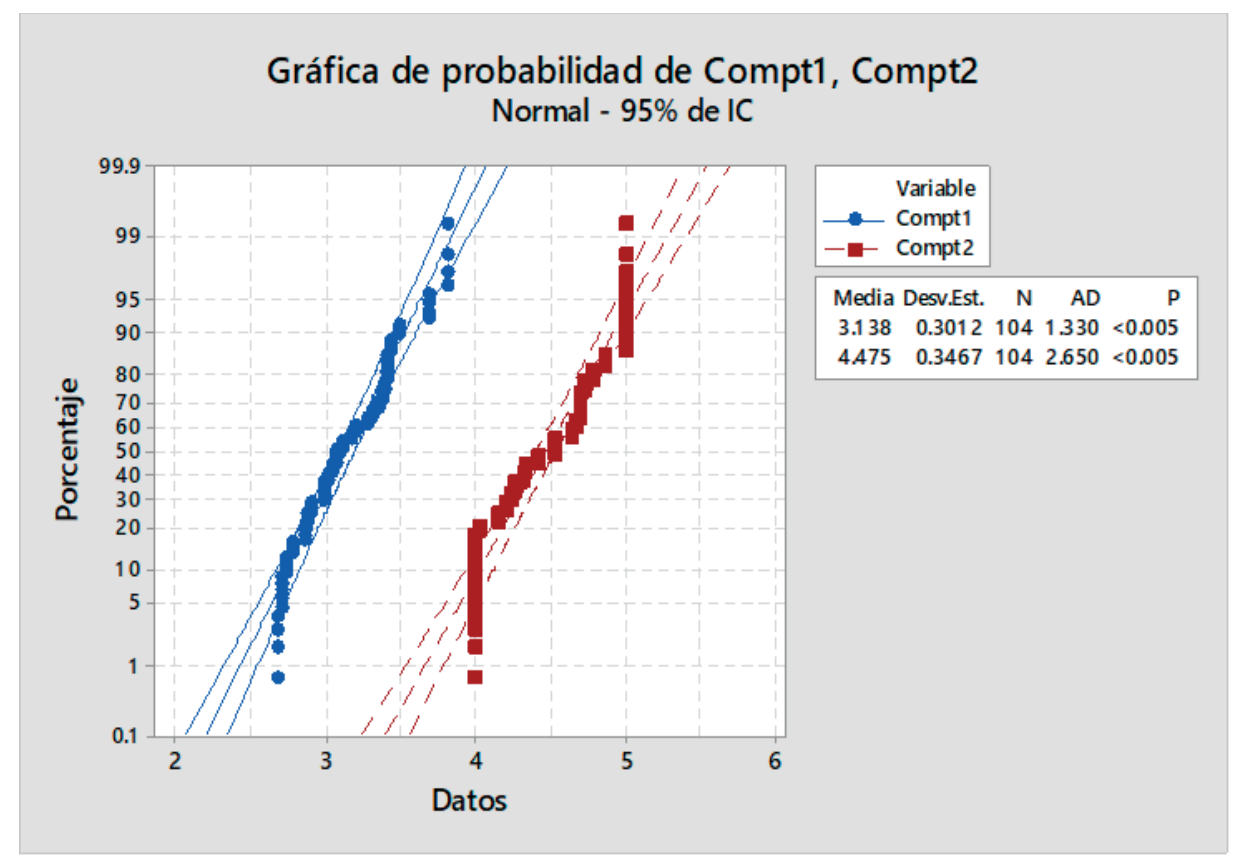

Figura 2. Comparación de probabilidad de la variable competitividad.

Fuente: Elaboración propia.

información estadística a favor de estas hipótesis. El objetivo estratégico de desarrollar al OMA-013 de la MGP como una EPDP tiene como finalidad hacerla competitiva en el servicio de mantenimiento aeronáutico en el Perú en beneficio del Estado y la nación en su conjunto.

Producto del contraste, se encontró evidencia positiva para la denominada hipótesis alternativa $\left(\mathrm{H}_{1}\right)$, en la que, al sustentarla estadísticamente, existe una significativa mejora en las expectativas del recurso humano especializado del OMA-013 para con la afirmación de la hipótesis principal $\left(\mathrm{H}_{\mathrm{p}}\right)$, por lo cual la hipótesis nula $\left(\mathrm{H}_{0}\right)$ queda rechazada $(p<0.05)$ debido a la superior media de la evaluación postest con respecto al pretest en los resultados del instrumento de medición validado en la investigación.

En la tabla 5 se presenta la conclusión de la correlación identificada por el análisis estadístico aplicado a cada hipótesis específica y el impacto a sus respectivas variables dependientes, de manera que sus respectivas hipótesis nulas $\left(\mathrm{H}_{0}\right)$ queden rechazadas por el grado de significancia $(p<0.05)$ que resultó entre cada correlación efectuada en ambos eventos: 1. Evaluación pretest y 2. Evaluación postest.

\section{Propuesta para la solución del problema}

Planteado y evaluado el problema de la competitividad del OMA-013 en un marco legal no favorable, se presenta como solución del problema a una
OMA competitiva y autosostenible, que beneficie y satisfaga las necesidades aeronavales de la MGP en base y en función de un plan estratégico en el que se determinen claramente objetivos estratégicos que permitan:

- Desarrollar una EPDP en la MGP, con la finalidad de ser más competitiva.

- Implementar y capacitar el Capability DGAC que le genere mayor competitividad.

- Lograr, en función al Capability del OMA013, la obtención de una autosostenibilidad financiera, en beneficio del sector aeronáutico en la MGP y del país.

- Generar actividades rentables a través del conocimiento de las limitaciones iniciales del actual presupuesto asignado para el funcionamiento de la capacidad instalada del OMA-013.

- Aprovechar la influencia y el marco legal que genera el SIMA Perú para las estrategias de competitividad que se desarrollen en Capability del OMA-013.

\section{CONCLUSIONES Y RECOMENDACIONES}

Los recursos humanos de una organización de mantenimiento aeronáutico certificada por la Dirección General de Aeronáutica Civil (DGAC) son 
Tabla 5. Correlación de las variables del OMA-13 y las hipótesis de la investigación.

\begin{tabular}{|c|c|c|c|c|c|c|c|c|}
\hline \multirow{3}{*}{\multicolumn{2}{|c|}{ HIPÓTESIS }} & \multirow{2}{*}{\multicolumn{3}{|c|}{$\begin{array}{c}\text { Variable Independiente } \\
\text { Dimensiones }\end{array}$}} & \multicolumn{4}{|c|}{ Variable Dependiente } \\
\hline & & & & & \multicolumn{4}{|c|}{ Dimensiones } \\
\hline & & $\begin{array}{c}\text { Gestión } \\
\text { mant. }\end{array}$ & $\begin{array}{c}\text { Cultura } \\
\text { organizacional }\end{array}$ & $\begin{array}{l}\text { Capability } \\
\text { TMA }\end{array}$ & Rentabilidad & $\begin{array}{l}\text { Agilidad } \\
\text { comercial }\end{array}$ & $\begin{array}{c}\text { Aseguramiento } \\
\text { calidad }\end{array}$ & Tecnología \\
\hline \multirow{3}{*}{$\mathrm{H}_{\mathrm{p}}$} & \multirow{3}{*}{$\begin{array}{l}\text { Independiente: P0. Desarrollo } \\
\text { OMA013: Xp } \\
\text { Dependiente: P0. Competitividad: } \\
\text { f (x) } \\
\text { Interviniente: P0. RR. HH. y RR. } \\
\text { MM. asignado }\end{array}$} & & & & & & & \\
\hline & & & & & & & & \\
\hline & & & & & & & & \\
\hline \multirow{3}{*}{$\mathrm{H}_{\mathrm{s}} 1$} & \multirow{3}{*}{$\begin{array}{l}\text { Independiente: 1. Capability } \\
\text { OMA013: Xp1 } \\
\text { Dependiente: } 1 \text {. Mejor competitivi- } \\
\text { dad: } f(x 1) \\
\text { Interviniente: 1. RR. HH. y RR. MM. } \\
\text { asignado } \\
\end{array}$} & & & & & & & \\
\hline & & & & & & & & \\
\hline & & & & & & & & \\
\hline \multirow{3}{*}{$\mathrm{H}_{\mathrm{s}} 2$} & \multirow{3}{*}{$\begin{array}{l}\text { Independiente: 2. Capability Prior. } \\
\text { OMA013:Xp2 } \\
\text { Dependiente: } 2 \text {. Estados Financie- } \\
\text { ros: } f(x 2) \\
\text { Interviniente: } 1 \text {. RR. HH. y RR. MM. } \\
\text { asignado }\end{array}$} & & & & & & & \\
\hline & & & & & & & & \\
\hline & & & & & & & & \\
\hline \multirow{3}{*}{$\mathrm{H}_{\mathrm{s}} 3$} & $\begin{array}{l}\text { Independiente: 3. Instalaciones } \\
\text { OMA013:Xp3 }\end{array}$ & & & & & & & \\
\hline & Dependiente:3. Planificación: f (x3) & & & & & & & \\
\hline & $\begin{array}{l}\text { Interviniente: 1. RR. HH. y RR. MM. } \\
\text { asignado }\end{array}$ & & & & & & & \\
\hline \multirow{3}{*}{$\mathrm{H}_{\mathrm{s}} 4$} & \multirow{3}{*}{\begin{tabular}{|l} 
Independiente: 4. Marco Legal \\
OMA013:Xp4 \\
Dependiente: 4. Estrategia Compe- \\
titiva: $\mathrm{f}(\mathrm{x})$ \\
Interviniente: 1. RR. HH. y RR. MM. \\
asignado \\
\end{tabular}} & & & & & & & \\
\hline & & & & & & & & \\
\hline & & & & & & & & \\
\hline
\end{tabular}

Fuente: Elaboración propia.

imprescindibles en el proceso de mantenimiento de las aeronaves, por lo que el OMA-013 debe ser escrupulosa con la certificación del personal con cursos del fabricante (OEM - Original Equipment Manufacturer) o equivalentes.

El OMA-013 deberá certificar los TMA requeridos por la MGP de 1er, 2do y 3er nivel según sea el caso, aprovechando su capacidad instalada, personal especialista certificado y ubicación estratégica al brindar estos servicios de mantenimiento a aquellas actividades y entidades con mayor demanda comercial, de manera que se genere el financiamiento adecuado que le permita resolver el problema administrativo de su organización, y en el mediano/largo plazo, convertirse en una organización rentable y autosostenible.

Basados en los cuatro pilares para la gestión de mantenimiento de una OMA, el OMA-013 deberá certificar los TMA con mayor demanda comercial requeridos por la MGP, de acuerdo a su estudio de mercado y a las estadísticas de su experiencia en el sector y su visión estratégica en el diseño de sus hangares, que puedan generar recursos financieros que permitan resolver el problema técnico de las certificaciones de sus TMA y como consecuencia, que estos permitan al OMA-013 gestionarse eficientemente y lograr rentabilidad y autosostenibilidad.

El Servicio de Mantenimiento Aeronaval deberá emplear herramientas administrativas y financieras para aprovechar y gestionar su infraestructura, recurso humano y ubicación estratégica, a fin de preparar el entorno adecuado que le permita gestionar ante el Almirantazgo de la MGP la autorización al OMA-013 de operar como una Empresa Pública de Derecho Privado y se realicen todas las gestiones necesarias que permitan tal funcionamiento, teniendo presente la focalización en lograr rentabilidad y autosostenimiento.

EI OMA-013 deberá efectuar las gestiones necesarias que le permitan convertirse en una EPDP en el corto plazo, aprovechando el marco legal con el que realiza sus actividades el SIMA Callao, de 
manera que pueda desempeñarse como una actividad más de negocios de este arsenal naval con sede en Lima, Perú, manteniendo la actual cultura organizacional del OMA-013 como organización aeronáutica, la cual es regulada por la Dirección General de Aviación Civil en el Perú (DGCA), así como por normas y costumbres de la actividad aeronáutica mundial que influencia al Perú. El sector aeronáutico exige especial cuidado por el entorno de riesgo en el que se desenvuelve, que lo hace particular y específico en procedimientos y trazabilidad.

El plan estratégico propuesto se debe poner en práctica, comprendiendo e interiorizando todas las estrategias y objetivos formulados, los cuales tendrán que ser valorados pragmáticamente antes de su ejecución y control durante la gestión. El plan deberá empezar por lo que le genere mayor rentabilidad y sea alcanzable rápidamente en el corto plazo con la finalidad de mejorar su situación actual, a fin de aspirar a cumplir con los objetivos del mediano y largo plazo, según la misión y visión de la organización, cambiando y ampliando las estrategias y tácticas que le permitan mantener vigente la implementación de mejoras que estén alineadas a su misión y objetivos planteados.

\section{AGRADECIMIENTOS}

A mis profesores, asesores, profesionales y personas especiales que me apoyaron y acompañaron durante la formulación de la presente tesis. A mis padres, esposa e hijos, quienes se privaron de muchas actividades importantes por mi constante ausencia, y a mis amigos y compañeros de profesión: todos ellos fomentaron mi perseverancia en concretar con éxito la obtención del grado académico.

\section{REFERENCIAS BIBLIOGRÁFICAS}

[1] Amendola, L., Depool. T., y Castillo, M. (2016). Impacto de los Capex y Opex en la Gestión de Activos. Recuperado de https://www.linkedin. com/pulse/impacto-de-los-capex-y-opex-en-lagesti\%C3\%B3n-activos-amendola/

[2] Blanchard, K., Randolph, W., y Grazier P. (2006). Trabajo en Equipo: Go Team! Tres pasos para conseguir grandes resultados. Barcelona, España: Deusto.

[3] Constitución Política del Perú [Const.] Art. 60, 29 de diciembre de 1993.

[4] Crespo, A (2007). The Maintenance Management Framework: Models and Methods for Complex Systems Maintenance. Londres, Reino Unido: Springer.
[5] Davenport, T. (2006). Capital Humano. Barcelona, España: Deusto

[6] D. L. N 1031. Decreto Legislativo que promueve la eficiencia de la actividad empresarial del Estado. Ministerio de Economía y Finanzas. Recuperado de https://www.mef.gob.pe/es/ por-instrumento/decreto-legislativo/6558decreto-legislativo-n-1031/file

[7] Hamel, G., Doz, Y., Bettis, A., y Prahalad, C. (2006). Estrategia corporativa. Barcelona, España: Deusto

[8] Ley $N^{\circ} 24948$ (1988, 2 de diciembre). Ley de la Actividad Empresarial del Estado. Ministerio de Economía y Finanzas. Recuperado de https:// www.grupoconsultorefe.com/assets/files/ recursos/files/Per\%C3\%BA\%20-\%20Ley\%20 N\%C2\%BA\%2024948_4287.pdf

[9] Mateo, R. (2015). Propuesta y validación de un modelo integrador de Implantación del Mantenimiento Productivo Total (TPM), Aplicación en una empresa industrial. (Tesis doctoral). Universidad de Valencia, Valencia.

[10] Porter, M. (2006). Estrategia y ventaja competitiva. Barcelona, España: Deusto

[11] Riquelme, M. (2016). Matriz o Análisis FODA - Una herramienta esencial para el estudio de la empresa. Recuperado de https://www. analisisfoda.com/

[12] Servicio Industrial de la Marina de Guerra del Perú-SIMA (2020). Historia del Sima (25 de marzo de 1999). Recuperado el 4 de diciembre de 2020 de http://www.sima.com.pe/

[13] Villalón, M. (2015). Gestión y vinculación para un alto desempeño de la investigación en aeronáutica. (Tesis de Doctorado). Universidad de Querétaro, Querétaro.

[14] Vogtlande, J., Scheepens, A., Bocken, N., y Peck, D. (2017). Combined analyses of costs, market value and eco-costs in circular business models: eco-efficient value creation in remanufacturing. Journal of Remanufacturing. 7(1), 1-17. Recuperado de https://doi. org/10.1007/s13243-017-0031-9 


\section{Development of State-Owned Enterprises Subject to Private Law in the Aeronautical Maintenance Sector in the Peruvian Navy}

CÉSAR Angulo Rebaza ${ }^{1}$

Received: 11/11/2020 Accepted: 19/01/2021 Published: 26/07/2021

\begin{abstract}
The investigation consisted of demonstrating methodologically that it is possible to develop the aeronautical maintenance industry within the Peruvian Navy (MGP) by means of a novel undertaking in the field of business management within the MGP, that is to say, the design of a state-owned enterprise (SOE) subject to private law that allows the growth of the aeronautical sector within a legal framework that helps achieve a balance between efficiency and effectiveness. The analysis of the current situation of aeronautical maintenance in the MGP identified that it is not taking advantage of potentialities in human and certified materials (capability). Therefore, the application of administrative theories will make it possible to optimize the management process, generating greater competitiveness and profitability. The correlational descriptive method was used through the application of surveys and its results validated the proposal, as well as its viability as a decentralized and specialized agency of SIMA Peru. This scenario would contribute to meeting the aeronautical needs of the MGP in maintenance management practices, organizational culture, TMA capability, profitability, commercial agility, technology and quality assurance, which have a significant relationship with operational performance, that also impacts the development of aeronautical technology in the country.
\end{abstract}

Keywords: aeronautical maintenance; profitability; competitiveness; capability.

\section{INTRODUCTION}

Competitiveness represents a constant business challenge in the search for effectiveness and efficiency, which is why governmental and private entities need to restructure themselves administratively and financially so they do not lose momentum. This is the case of the Aeronaval Maintenance Service of the Marina de Guerra del Perú5 (MGP), certified by the Dirección General de Aeronáutica $\mathrm{Civil}^{6}$ (DGAC) as "Organización de Mantenimiento Aprobada (OMA-013): Servicio de Mantenimiento Aeronaval"7, which operates commercially, propelled by the capacity installed, certified and financed with the funds contributed by MGP according to their availability, which has not been sufficient neither to achieve its technical-administrative self-sustainability, much less fulfill its mission of maintaining the Naval Aviation Force fleet. Therefore, this study proposes the development of an innovative state-owned business management within the framework of private law that will contribute to the fulfillment of the mission and aeronautical development of the MGP.

OMA-013 is the technical, repair and maintenance body of the highest aeronautical level in the MGP, whose objective is to execute manufacturer scheduled maintenance (second level) and component repairs (third level) to the aircraft assigned to the Naval Aviation Force and to aircraft and accessories authorized in commercial aviation. This aeronautical maintenance organization has been working with budgetary restrictions for three decades and with some non-constant and almost null representative investments over the last five years, which has produced negative effects within the maintenance process, worsened further by the lack of an adequate administrative management.

The optimal functioning of OMA-013 is currently being affected by the increasing updating of international aeronautical requirements by the DGAC, which decreases its competitiveness due to the decreasing certified technical capacity of its human and ma-

\footnotetext{
Master in Aeronautical Engineering from Universidad Nacional de Ingeniería and Master in Business Administration from Universidad Nacional Agraria La Molina (Lima, Peru). Currently working as a maritime pilot and aeronautical engineer of the Peruvian Navy, in operational, technical and administrative positions. Experience in leading public and private investment projects, educational management and industrial management (Lima, Peru). ORCID: https://orcid.org/0000-0002-5261-5598

Corresponding author: cesar.angulo.rebaza.21@gmail.com

5 Peruvian Navy.

6 General Directorate for Civil Aviation.

7 Approved Maintenance Organization (OMA-013): Aeronaval Maintenance Service.
} 
terial resources (capability) of its aeronautical maintenance workshops (TMA). This unexploited technological space is detrimental to the development of the aeronautical sector in the MGP, which has national repercussions $\mathrm{n}$, since this activity has not yet found a significant commercial development. On the contrary, the majority (more than $80 \%$ ) of the current and important repairs are made outside the country in foreign entities dedicated to aeronautical engineering activity, which are not yet fully established in the country.

The manifest problem of OMA-013 is in an increasingly critical situation, where the necessary administrative theories and management tools cannot be applied, which gives rise to the following question: How to develop a competitive state-owned enterprise subject to private law in the area of aeronautical maintenance service in the MGP within Peru?

In response to this question, this research analyzes holistically the following characteristics of the organization: infrastructure and human resources (DGAC capability), profitability and legal framework. It also analyzes the management theories that can be applied in order to contribute to make OMA-013 more competitive in aeronautical maintenance service.

In order to identify the real conditions of OMA-013 and to formulate improvement plans, it is necessary to consider the contribution of several authors regarding the phenomena that affect organizations.

\section{Theoretical Framework}

Hamel et al. (2006) state that knowledge is fundamental for the economic progress of a country since it improves the performance of its organizations. Entrepreneurship internalizes concepts of information societies, knowledge societies, knowledge workers and information systems with the purpose of generating explicit and tacit knowledge. In addition, they emphasize human capital as a concept, which they recognize as the most valuable resource for an organization or company.

Porter (2006) states that "El campo competitivo es vital para la ventaja competitiva ya que configura la estructura de la cadena de valor, la forma en que se realizan las actividades y la posibilidad de que esas actividades sean compartidas por las unidades de la empresa" [the competitive field is vital for competitive advantage since it configures the structure of the value chain, the way in which activities are performed and the possibility that these activities are shared by the business units] (p.44). He adds:
La Estrategia Tecnológica representa entonces para una empresa la política en la que esta decide su desarrollo, mediante el empleo positivo de la tecnología y el poder que tiene el cambio tecnológico para con la estructura del sector industrial en general y aeronáutico en particular, asegurando su ventaja competitiva [For a company, the Technological Strategy represents the policy in which it decides its development, through the positive use of technology and the power that technological change has for the structure of the industrial sector in general and the aeronautical in particular, ensuring its competitive advantage]. (p.133)

Organizational culture generates union and direction among workers; therefore, teamwork ensures the success of any company or organization:

El trabajo en equipo es la capacidad de funcionar con éxito en un entorno de equipo, se está trasladando rápidamente a la primera línea de las habilidades empresariales necesarias (...) La gente que trabaja en grupos operativos, equipos de resolución de problemas, equipos de desarrollo de productos y comités aportan una visión colectiva que multiplica de forma importante su capacidad de innovar [Teamwork is the ability to function successfully in a team environment, which is rapidly moving to the front line of the necessary business skills (...) People working in operational groups, troubleshooting teams, product development teams and committees bring a collective vision that significantly multiplies their ability to innovate]. (Blanchard et al., 2006, pp. 70-71)

The work of Vogtlande et al. (2017) analyzes new techniques in the field of aeronautical engineering and evaluates combined cost, market value and ecocost analyses, which are the basis for eco-efficient value creation in remanufacturing. These authors argue that aeronautical remanufacturing processes will improve the sales of aircraft previously defined as successful by users in addition to generating added value, new useful life and new technologies.

Remanufacturing is the process of refurbishing a successful product by the manufacturer to bring it up to the level of a new product. This technology could be exploited by the aeronautical maintenance workshops of the MGP as it represents low costs in skilled labor compared to developed countries. In addition, it could represent a leap in the 
modernization of aeronautical technology in Peru, strengthen the installed capacity of the MGP and provide an opportunity for self-financing.

Davenport (2006) observes and analyzes human capital and its influence on competitive strategy. $\mathrm{He}$ points out that workers represent the differentiation of the company and its value in the market in more than $60 \%$ of its value, which highlights the intangibility of human capital, which is much more difficult to find than financial capital.

According to Crespo (2007), the maintenance management process conditions effectiveness and efficiency in management. The theory reviewed suggests that the effectiveness of business activities is framed in terms of capability, profitability, commercial agility, technology and service quality from the customer perspective. Efficiency, always followed by effectiveness, ensures the production with no waste of resources, which minimizes direct maintenance costs, and maintenance, in turn, becomes more competitive.

Amendola et al. (2015) published in an open source their conclusions on asset management. They state that the competitiveness of companies is characterized by their constant budget cuts; by the efficiency of their goods or services in accordance to high quality standards; by the alignment to their strategic plan; by the total expenditure (TOTEX); by theories of capital expenditure (CAPEX), which is used according to the management of physical assets in the actual operational activity of the company; and, finally, by their management of operational expenditure (OPEX), which defines the costs associated with the exploitation of these physical assets.

Hamel et al. (2006) point out that it is necessary to develop a "strategic control" in the center of power and its respective areas of influence (subsidiaries). They consider that some typical strategic decisions are technology, market definition of products, emphasis on the different product lines, expansion and diversification of operations, and the desire to participate in a global network of product flows between the areas of influence or subsidiaries. In that sense, the headquarters should not only be interested in controlling and influencing all strategic decisions, but also in monitoring their progress towards the fulfillment of strategic expectations.

\section{Legal Framework}

The legal framework of this research was based on national regulations that are the formalization and legal foundation proposed in this article.
Regarding the business activity in Peru, Law No. 24948 (1988, December 2) determines that stateowned enterprises, state-owned enterprises subject to private law, mixed economy companies and the State's stockholders are public sector entities, in accordance with the provisions of article 60 of the Political Constitution of Peru (1993) which recognizes economic pluralism based on the coexistence of different forms of enterprise and ownership. In article 1 , this law regulates the business activities of the State in terms of its scope; objective; organization; operation; labor, administrative, economic and financial regime; and evaluation and interaction at the different levels of government. Likewise, article 5 establishes that the State generates business activity in different forms, among which, for the purposes of this work, the following form of business, explained in article 7 , is highlighted:

- State-owned enterprises subject to private law: These are las constituidas originalmente o reorganizadas como sociedad anónima de acuerdo a ley, cuyo capital pertenece totalmente al Estado [those originally created or reorganized as a corporation according to law, whose capital belongs entirely to the State] (Act No. 24948, 1988).

Additionally, Legislative Decree No. 1031 promotes the efficiency of the business activity of the State by stating that it is required to implement nuevas herramientas de gestión y estructuras legales que prioricen los principios de eficiencia, transparencia y gobierno corporativo, entre otros, así como un sistema de control adecuado en un contexto de transparencia [new management tools and legal structures that prioritize the principles of efficiency, transparency and corporate governance, among others, as well as an adequate control system in a context of transparency] (Decreto Legislativo No. 1031, 2018).

The National Fund for the Financing of the Public Sector Companies (FONAFE) is the entity in charge of regulating the business activity of the State, ensuring that these companies are managed in a modern manner and according to recognized quality standards for their products (goods and/or services) so they generate economic value in favor of society and respect for the environment to achieve the sustained development of Peru, based on innovation and integration as specific corporate values.

According to the preceding paragraphs, it can be appreciated that the Peruvian State has the power to exercise business activity, directly or indirectly, under the same conditions as a private company, 
although with special characteristics based on its rules and regulations, in which a state-owned enterprise (SOE) subject to private law can do everything that is not legally prohibited in order to optimize its management processes, as suggested by Mateo (2015), whose doctoral thesis had as general objective to define maintenance management processes and the causes that affect success in development models.

\section{Servicios Industriales de la Marina, SIMA}

SIMA is a state-owned enterprise (SOE) subject to private law of FONAFE Corporation, set up as a joint stock company in 1999, created with the objective of promover $y$ desarrollar la industria naval, industrias complementarias y conexas [promoting and developing the naval industry, complementary and related industries], prioritizing the repair and construction of MGP ships (SIMA, 2000). It currently has the potential to propel the activity in the aeronaval field as a business line with the integration of the capability developed by OMA-013.

According to the SIMA portal (2020), the business activity of the State is regulated by Law No. 24948 (1988), which states in Article 6 that Son empresas de derecho público las que se crean por ley y gozan de atributos propios de la administración pública [State-owned enterprises are those created by law and enjoy the attributes of public administration], and in Article 7 states that Son empresas de Derecho Privado las constituidas originalmente o reorganizadas como sociedad anónima de acuerdo a ley, cuyo capital pertenece totalmente al Estado [State-owned enterprises subject to private law are those originally constituted or reorganized as a corporation according to law, whose capital belongs entirely to the State].

In that sense, the legal framework has been favorable for the development of SIMA and made it possible to have three operation centers located in Callao and its subsidiaries in Chimbote and Iquitos, the following being its three main lines of business:

- Preventive and corrective maintenance to ships, called naval repair.

- Construction of ships, called naval construction.

- Production of metallic structures (bridges, towers, hydroelectric gates, etc.), called metalmechanics.

In his doctoral thesis, Villalón (2015) concludes that it is necessary to develop and apply a large variety of quantitative and qualitative methods in the effective search to know the management competencies and bonding schemes that allow building a model that results in high performance. In this regard, it can be stated that SIMA is a company that specializes in naval engineering, hosts naval aeronautical activity in its naval platforms, participates in diverse and important technological activities, has excellent competencies for technical and business management, and has high levels of performance, unique in the nation, thus contributing to the development of the country.

For this reason, when analyzing the legal framework of the theory presented in this investigation, SOE subject to private law turns out to be a business management model characterized by longterm objectives, satisfaction and positive impact on the development of the country. This management model provides modern management tools that add reputation and value to any company before its stakeholders and incorporates them into management processes from a systemic perspective, which would be reflected in the generation of public and private value. This represents an enormous opportunity for business development in the aeronautical maintenance service in the search for social, technological, business, and internal and external defense development of the nation.

According to the theories presented in this study and the experience of the MGP in the aeronautical development of the country, this research proposes that the Aeronaval Maintenance Service OMA-13 would be successful as a SOE subject to private law if based on the legal framework of SIMA, as a subsidiary of the development of the technical capacity of the aeronaval environment, under its legal responsibility, without the need to create another entity to be managed as a SOE subject to private law. This would promote the development of technology in the country, since there is no such specialized and particular technological development in the naval or aeronaval field.

SWOT analysis of the variables according to their validated dimensions and their statistical results

In his illustration about strategic analysis and planning, Riquelme (2016) explains that the term SWOT is an acrostic of:

- Strengths: positive critical factors that are available;

- Weaknesses: negative critical factors that must be eliminated or reduced; 
- Opportunities: positive aspects that can be leveraged using the strengths; and

- Threats: external negative aspects that could hinder the achievement of our objectives.

The SWOT analysis is highly recommended since it is the stage prior to decision making. This simple and effective method is used to determine the real condition of a company regarding its environment, so a forward-looking strategy can be planned.

In order to develop a competitive SOE subject to private law for the benefit of the MGP and the country in the aeronautical sector, an analysis of strengths, weaknesses, opportunities and threats (SWOT) was carried out in accordance with the theory presented, which determined qualitatively and according to quantitative weights the strategies to be chosen according to strategic objectives to be fulfilled (strategic decision). Based on field work and the experience in the management of this maintenance organization, it is relevant to mention that this analysis presented the real situation of OMA-013, which was said to be stuck in an "area of vulnerability".

The SWOT analysis showed an OMA-013 incapable of self-sustainability, fundamentally as it is not competitive in the aeronautical sector, with the main weakness being the time it takes to get client aircraft on the flight line and operational; the latter happens due to the rules of the public administration in which it operates, which is aggravated by the repercussions of the financial resource available to achieve the capability standards required and regulated by the DGAC.

Table 1 shows a SWOT matrix for OMA-013. This approach will provide management with very important elements to build a strategy for the future that will allow the development of concrete actions focused on improving competitiveness and market position.

Based on this reality, the management direction of OMA-013 was proposed as a SOE subject to private law through long-term strategies, taking into consideration the verification of the survey proposed in the research, which determined the strategies to be implemented in the Aeronaval Maintenance Service according to the values obtained and based on the objectives described in Table 2.

The internal and external analysis of the environment in which the activities of OMA-013 develop concludes that strategies are required to redefine its activity as a SOE subject to private law in order to obtain greater competitiveness in the national market and to ensure that OMA-013 stops being in an area of vulnerability in that it is unable neither to fulfill its main mission nor to achieve financial self-sustainability requirements of the MGP and the nation in the aeronautical sector.

Table 1. SWOT Matrix for OMA-013.

\begin{tabular}{|c|c|}
\hline INTERNAL FACTORS & EXTERNAL FACTORS \\
\hline $\begin{array}{l}\text { STRENGHTS } \\
\text { - } \text { Certified HR in the service. } \\
\text { - } \quad \text { Contract renewal of certified HR. } \\
\text { - } \quad \text { Increase of medium and large customers. } \\
\text { - } \quad \text { Differentiated installed capacity. } \\
\text { - } \quad \text { Certainty of increasing demand. }\end{array}$ & $\begin{array}{l}\text { OPPORTUNITIES } \\
\text { - Increase of service orders and associated budget. } \\
\text { - Lack of aeronautical maintenance workshops in the } \\
\text { market. } \\
\text { - Increase of TMA capabilities (more service orders). } \\
\text { - Reliable logistics providers. }\end{array}$ \\
\hline $\begin{array}{l}\text { WEAKNESSES } \\
\text { - HR and technological upgrade costs. } \\
\text { - } \quad \text { Continuous investment in capability. } \\
\text { - } \quad \text { Medium capacity to retain the HR. } \\
\text { - } \quad \text { Low marketing aggressiveness. } \\
\text { - } \quad \text { Lack of incentives for initial HR. }\end{array}$ & $\begin{array}{l}\text { THREATS } \\
\text { - } \quad \text { Appearance of more competition. } \\
\text { - } \quad \text { Strengthening of other TMAs of similar function. } \\
\text { - } \quad \text { Unreliable logistics providers. } \\
\text { - } \quad \text { Retention capacity of initial HR-CG }\end{array}$ \\
\hline
\end{tabular}

Source: Prepared by the author.

5 General Command: The generic specialty that the human resource starts with in the MGP. 
Table 2. Strategies and objectives proposed on the research.

\begin{tabular}{|l|l|}
\hline Variables & Objective/Strategy \\
\hline \multirow{2}{*}{$\begin{array}{l}\text { Independent: } \\
\text { Development of the Capability of OMA-13 }\end{array}$} & Maintenance management (UNE-EN 15341) \\
\cline { 2 - 2 } & Organizational culture \\
\cline { 2 - 2 } & TMA capability \\
\hline \multirow{4}{*}{$\begin{array}{l}\text { Dependent: } \\
\text { Competitiveness }\end{array}$} & Profitability \\
\cline { 2 - 2 } & Commercial agility \\
\cline { 2 - 2 } & Quality assurance \\
\cline { 2 - 2 } & Technology \\
\hline
\end{tabular}

Source: Prepared by the author.

\section{METHODOLOGY}

This was a mixed descriptive correlational research of cross-sectional observational type, which seeks to determine the degree of relationship existing between the variables.

According to the field research carried out in the aeronautical maintenance workshops of the Aeronautical Maintenance Service (OMA-013) of the Peruvian Navy, the following methodological aspects were considered: research design, variables, population, instruments, data collection tools and data analysis.

In that sense, the research was corroborated by qualitative data, which was the product of a Likert scale survey, so that the inference could be measurable according to the variables established in the study. This analysis is related to inductive methods that obtain narrative records of the phenomena studied through direct observation techniques, interviews, field notes and surveys to the population of interest.

The sample size covers 104 people dedicated to the technical and administrative management of 10 aeronautical maintenance workshops certified by the MGP before the General Directorate of Civil Aviation. Due to the constant military rotation, the number of people was higher than the minimum determined in the sample, so that any eventuality that would alter the study could be covered, that is, in order to obtain enough information to apply statistical-descriptive techniques to the people who manage the OMA-013. The minimum number of people to be surveyed was established at $95 \%$ reliability.

\section{RESULTS AND DISCUSSION}

\section{Statistical results of the Aeronaval Maintenance Service OMA-013}

The measurement instrument used was the questionnaire, which was applied to the sample control group, constituted by managers, chief officers, junior officers and workshop chiefs of OMA-013. After obtaining the results and their validation, the corresponding analysis was carried out to determine the dimensions in which the dependent and independent variables are developed; the results are presented below.

\section{Statistical Contrast of the Main Hypothesis $\left(\mathrm{H}_{\mathrm{p}}\right)$}

In order to analyze and present the statistical contrast of the main hypothesis $\left(\mathrm{H}_{\mathrm{p}}\right)$, "Developing OMA013 as a SOE subject to private law in the Peruvian Navy (MGP) will make it competitive in the aeronautical maintenance service in Peru", the confidence interval and the significant differences between the variances of the means to be contrasted (pretest and posttest) were calculated by ANOVA. Then the differences of the values of the calculated means were statistically compared, according to the main hypothesis and its secondary hypotheses $\left(\mathrm{H}_{\mathrm{s}}\right)$ in the 2 moments, pretest and posttest, to which they correspond. The result of the field work process was the conviction of the importance of the specialized human resource of OMA-013, with which the independent variables were worked, resulting in a positive difference in the dependent variables in the posttest.

The $\mathrm{H}_{\mathrm{p}}$ shows an independent variable characterized by three dimensions: maintenance management, organizational culture and capability of the aeronautical maintenance workshops. By improving their management as SOE subject to private law, those dimensions influence the competitiveness of the OMA-013 in the aeronautical sector. The dependent variable is characterized in four dimensions: profitability, commercial agility, quality assurance and technology.

The pre-test and post-test surveys made it possible to obtain important information on how staff perceives the performance of OMA-013 and, based on 
that, to propose alternatives to enhance installed capacity.

In that sense, the weights obtained from the pretest (Table 3 ) and post-test (Table 4) surveys were evaluated, paying special attention to the degree of significance at $95 \%$ for this research $(p<0.05)$ in order to prove true the main hypothesis $\left(\mathrm{H}_{\mathrm{p}} / \mathrm{H}_{1}\right)$.

\section{Significance by magnitude of the difference be- tween means}

The comparison of means of the dependent variable is shown in Figure 1 where a clear difference can be seen between the first stage of the study and the second one. The increase in the post-test moment is due to the OMA-013 population group to which was applied the validated measurement instrument. The variables for maintenance management (profitability, business agility, quality assurance and technology) within an adequate legal framework (SOE subject to private law) were fully identified as a propelling determinant of the business success of this MGP Maintenance Organization.

The difference of means between pre-test and posttest shows typical standard deviations due to their differences. In the sample $t$ analysis appears the most important statistical data to corroborate the hypothesis and its variables, that is, the degree of significance $(p<0.05)$, by which it is concluded that the characteristics of the SOE subject to private law used for the development of the OMA-013 will result in greater competitiveness in the aeronautical sector and, in consequence, capacity of self-sustainability and profitability after finding in the posttest a positive increase in the weighting versus the values that were reached in the pre-test, which can be seen in Figure 2.

According to the results of the SWOT analysis and the statistical analysis carried out in the investigation,

Table 3. Dependent variable "Competitiveness": Pre-test average data.

\begin{tabular}{|l|c|c|c|c|c|c|}
\hline Dependent V. & N & Mean & Std. Dev. & Q1 & Median & Q3 \\
\hline Competitiveness & 104 & 3.1524 & 0.602 & 2.8148 & 3.0926 & 3.4074 \\
\hline
\end{tabular}

$\mathrm{N}$ : Sample size.

Source: Prepared by the author.

Table 4. Dependent variable "Competitiveness": Post-test average data.

\begin{tabular}{|c|c|c|c|c|c|c|}
\hline Dependent V. & N & Mean & Std. Dev. & Q1 & Median & Q3 \\
\hline Competitiveness & 104 & 4.5196 & 0.0062 & 4.5185 & 4.5185 & 4.5185 \\
\hline
\end{tabular}

Source: Prepared by the author.

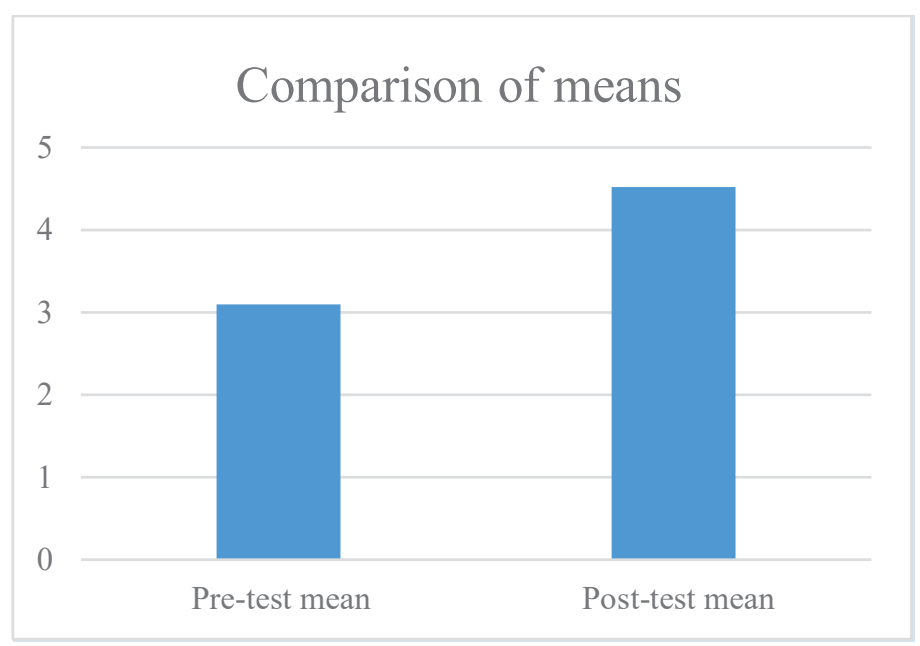

Figure 1. Comparison of means of the dependent variable. Source: Prepared by the author. 


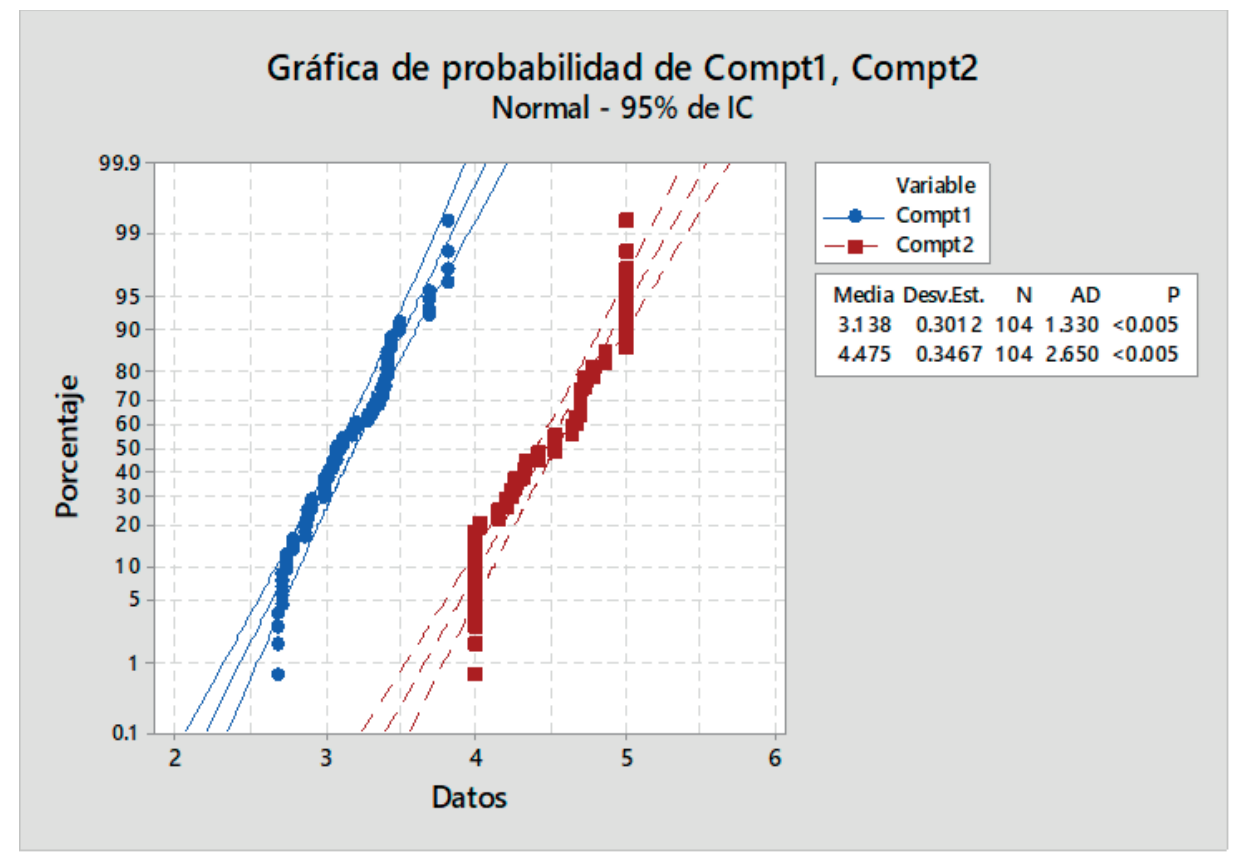

Figure 2. Comparison of Probability of Competitiveness Variable. Source: Prepared by the author.

the hypotheses proposed as guides of the investigation have been supported by a methodologically demonstrated truth, which makes possible the determination of strategic objectives in a management plan that influences positively in improving the reality of the aeronautical sector in Peru. The hypotheses proposed in the research received empirical evidence, which were weighted according to the evaluated phenomenon, showing statistical information in favor of these hypotheses. The strategic objective of developing OMA-013 of the MGP as a SOE subject to private law aims to make it competitive in the aeronautical maintenance service in Peru for the benefit of the State and the nation as a whole.

As a result of the contrast, positive evidence was found for the so-called alternative hypothesis $\left(\mathrm{H}_{1}\right)$, which, when statistically supported, shows a significant improvement in the expectations of the specialized human resources of OMA-013 in relation to the statement of the main hypothesis $\left(\mathrm{H}_{\mathrm{p}}\right)$; thus, he null hypothesis $\left(\mathrm{H}_{0}\right)$ is rejected $(p<0.05)$ due to the higher mean obtained in the post-test evaluation in relation to the pre-test, according to the results of the measurement instrument validated in the research.

Table 5 presents the conclusion of the correlation identified by the statistical analysis applied to each specific hypothesis and the impact on their respective dependent variables, so that their respective null hypotheses $\left(\mathrm{H}_{0}\right)$ are rejected by the degree of significance $(p<0.05)$ that resulted between each correlation performed in both events: 1. pre-test evaluation and 2. post-test evaluation.

\section{Proposal for the solution of the problem}

After being proposed and evaluated the problem of the competitiveness of OMA-013 in an unfavorable legal framework, the solution to the problem is presented as a competitive and self-sustainable OMA, which benefits and satisfies the aeronaval needs of the MGP based on and according to a strategic plan with clearly determined strategic objectives that will allow:

- Developing a SOE subject to private law in the MGP in order to be more competitive.

- Implementing and training the capability compliant with DGAC requirements to generate greater competitiveness.

- Achieving, based on the OMA-013 capability, the financial self-sustainability, for the benefit of the aeronautical sector in the MGP and the country.

- Generating profitable activities based on the knowledge of the initial limitations of the current budget allocated for the operation of the installed capacity of OMA-013. 
Table 5. Correlation of the variables of OMA-13 and the hypotheses of the research.

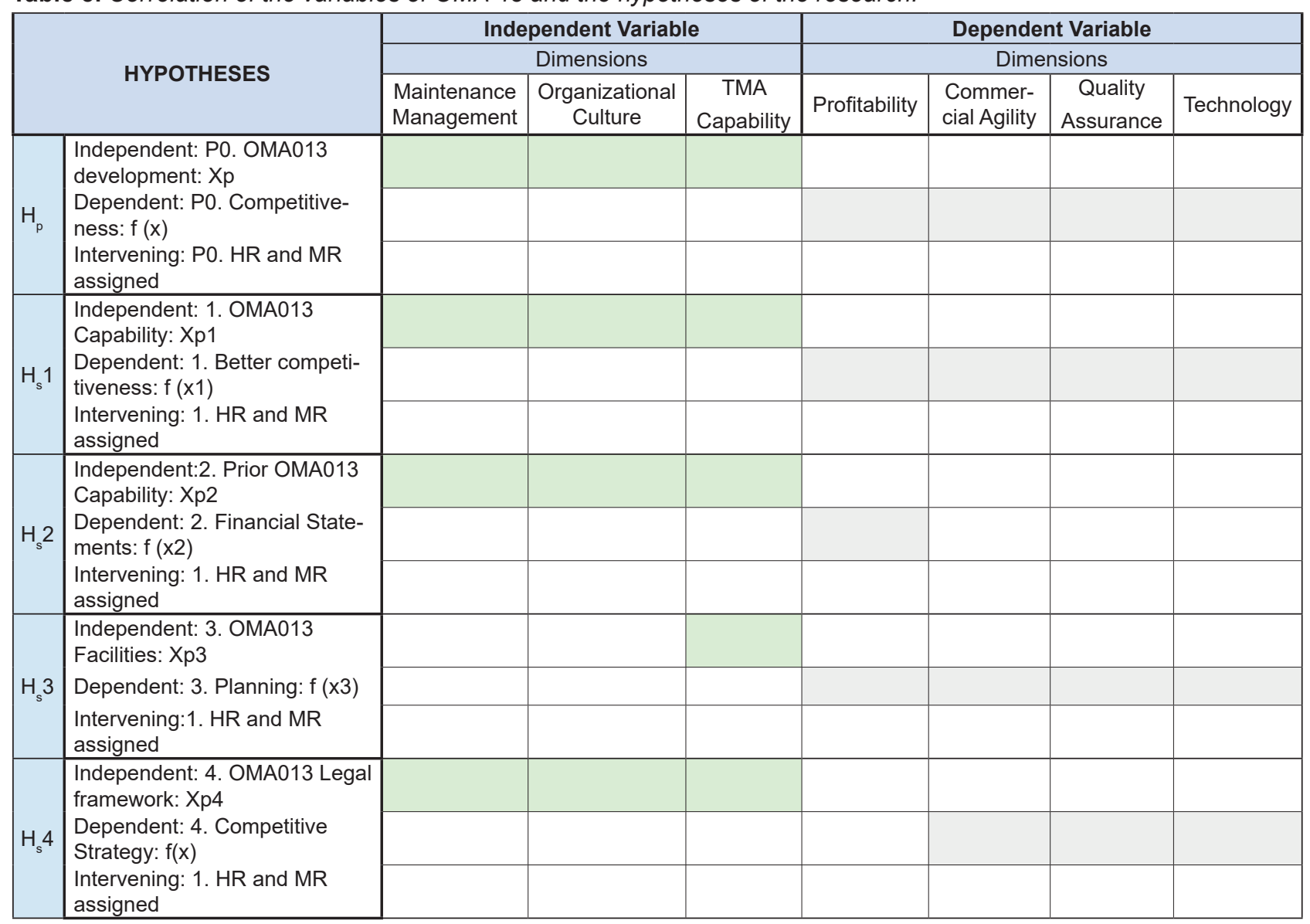

Source: Prepared by the author.

- Leveraging the influence and legal framework generated by SIMA Peru for the competitiveness strategies developed by OMA-013 capability.

\section{CONCLUSIONS AND RECOMMENDATIONS}

The human resources of an aeronautical maintenance organization certified by the General Directorate for Civil Aviation (DGAC) are essential in the process of aircraft maintenance, so OMA-013 must be scrupulous with the certification of personnel that have taken courses from the manufacturer (OEM Original Equipment Manufacturer) or equivalent.

OMA-013 must certify the TMAs of 1 st, 2 nd and 3rd level, as the case may be, required by the MGP, leveraging its installed capacity, certified specialist personnel and strategic location by providing these maintenance services to those activities and entities with higher commercial demand, so as to generate adequate funding that will allow it to solve the administrative problem of its organization, and be- come a profitable and self-sustainable organization in the medium/long term.

Based on the four pillars for the maintenance management of an OMA, the OMA-013 should certify the TMAs with the highest commercial demand required by the MGP, according to its market study and the statistics of its experience in the sector and its strategic vision in the design of its hangars, which can generate financial resources to solve the technical problem of the certifications of its TMAs and as a consequence, allow the OMA-013 to manage itself efficiently and achieve profitability and self-sustainability.

The Aeronaval Maintenance Service must use administrative and financial tools to manage and take advantage of its infrastructure, human resources and strategic location in order to prepare the adequate environment that will allow it to process, before the Admiralty of the MGP, the authorization for OMA-013 to operate as a state-owned enterprise subject to private law and to carry out all the necessary steps that 
will allow such operation, bearing in mind the focus on achieving profitability and self-sustainability.

OMA-013 must make the necessary arrangements to become a SOE subject to private law in the short term, taking advantage of the legal framework within which SIMA Callao carries out its activities, so that OMA-13 can perform another business activity of this naval arsenal based in Lima, Peru while maintaining the current organizational culture of OMA-013 as an aeronautical organization, regulated by the General Directorate of Civil Aviation in Peru (DGCA) and by the rules and customs of the world aeronautical activity that influences Peru. The aeronautical sector requires special care due to the risk environment in which it operates, which makes it particular and specific in terms of procedures and traceability.

The strategic plan proposed must be put into practice, comprehending and internalizing all the strategies and objectives formulated, which will have to be pragmatically evaluated before their execution and control during management. The plan must start by the items that generate the greatest profitability and are quickly achievable in the short term in order to improve the current situation and to meet the medium and long term objectives, according to the mission and vision of the organization, changing and expanding strategies and tactics that allow it to maintain the implementation of improvements that are aligned with its mission and objectives.

\section{ACKNOWLEDGEMENT}

To my professors, advisors, professionals and special people who supported and accompanied me during the formulation of my thesis. To my parents, wife and children, who were deprived of many important activities because of my constant absence, and to my friends and colleagues: all of them encouraged my perseverance in successfully obtaining my academic degree.

\section{REFERENCES}

[1] Amendola, L., Depool. T., \& Castillo, M. (2016). Impacto de los Capex y Opex en la Gestión de Activos. Retrieved from https://www.linkedin. com/pulse/impacto-de-los-capex-y-opex-en-lagesti\%C3\%B3n-activos-amendola/

[2] Blanchard, K., Randolph, W., \& Grazier P. (2006). Trabajo en Equipo: GoTeam! Tres pasos para conseguir grandes resultados. Barcelona, Spain: Deusto.
[3] Constitución Política del Perú [Const] Art. 2, 29 de diciembre de 1993.

[4] Crespo, A (2007). The Maintenance Management Framework: Models and Methods for Complex Systems Maintenance. London, United Kingdom: Springer.

[5] Davenport, T. (2006). Capital Humano. Barcelona, Spain: Deusto

[6] D.L. N. ${ }^{0}$ 1031.Decreto Legislativo que promueve la eficiencia de la actividad empresarial del Estado. Ministerio de Economía y Finanzas. Retrieved from https://www.mef.gob.pe/es/porinstrumento/decreto-legislativo/6558-decretolegislativo-n-1031/file

[7] Hamel, G., Doz, Y., Bettis, A., \& Prahalad, C. (2006). Estrategia corporativa. Barcelona, Spain: Deusto

[8] Ley $N^{\circ} 24948$ (1988, 2 de diciembre). Ley de la Actividad Empresarial del Estado. Ministerio de Economía y Finanzas. Retrieved from https:// www.grupoconsultorefe.com/assets/files/ recursos/files/Per\%C3\%BA\%20-\%20Ley\%20 N\%C2\%BA\%2024948_4287.pdf

[9] Mateo, R. (2015). Propuesta y validación de un modelo integrador de Implantación del Mantenimiento Productivo Total (TPM), Aplicación en una empresa industrial. (Doctoral thesis). Universidad de Valencia, Valencia.

[10] Porter, M. (2006). Estrategia y ventaja competitiva. Barcelona, Spain: Deusto

[11] Riquelme, M. (2016). Matriz o Análisis FODA - Una herramienta esencial para el estudio de la empresa. Retrieved from https://www. analisisfoda.com/

[12] Servicio Industrial de la Marina de Guerra del Perú-SIMA (2020). Historia del Sima (25 de marzo de 1999). Retrieved December 4, 2020, from http://www.sima.com.pe/

[13] Villalón, M. (2015). Gestión y vinculación para un alto desempeño de la investigación en aeronáutica. (Doctoral thesis). Universidad de Querétaro, Querétaro.

[14] Vogtlande, J., Scheepens, A., Bocken, N., \& Peck, D. (2017). Combined analyses of costs, market value and eco-costs in circular business models: eco-efficient value creation in remanufacturing. Journal of Remanufacturing. 7(1), 1-17. Retrieved from https://doi. org/10.1007/s13243-017-0031-9 